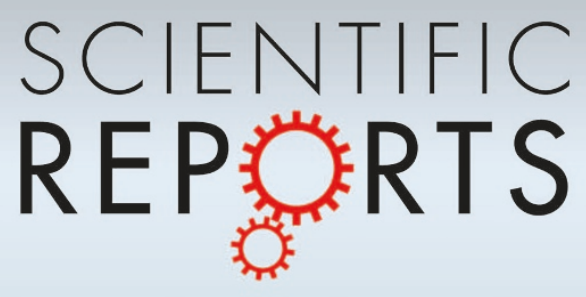

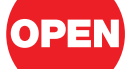

SUBJECT AREAS:

BIOLOGICAL PHYSICS

NONLINEAR PHENOMENA

SOCIAL EVOLUTION

CULTURAL EVOLUTION

Received

31 January 2013

Accepted

12 February 2013

Published

22 March 2013

Correspondence and requests for materials should be addressed to

F.C.S.

(franciscocsantos@ist.

utl.pt)

\section{Evolution of collective action in adaptive social structures}

\author{
João A. Moreira ${ }^{1,2}$, Jorge M. Pacheco ${ }^{1,3,4}$ \& Francisco C. Santos ${ }^{1,4,5}$
}

\begin{abstract}
${ }^{1}$ ATP-group, CMAF, Instituto para a Investigação Interdisciplinar, P-1649-003 Lisboa Codex, Portugal, ${ }^{2}$ Department of Chemical and Biological Engineering, Northwestern University, Evanston, Illinois, USA, ${ }^{3}$ Departamento de Matemática e Aplicações, Universidade do Minho, 4710-057 Braga, Portugal, ${ }^{4}$ Kavli Institute for Theoretical Physics, University of California Santa Barbara, CA 93 106-4030, USA, ${ }^{5}$ Departamento de Engenharia Informática \& INESC-ID, Instituto Superior Técnico, Universidade Técnica de Lisboa, IST-Tagusparque, 2744-016 Porto Salvo, Portugal.
\end{abstract}

Many problems in nature can be conveniently framed as a problem of evolution of collective cooperative behaviour, often modelled resorting to the tools of evolutionary game theory in well-mixed populations, combined with an appropriate $\mathrm{N}$-person dilemma. Yet, the well-mixed assumption fails to describe the population dynamics whenever individuals have a say in deciding which groups they will participate. Here we propose a simple model in which dynamical group formation is described as a result of a topological evolution of a social network of interactions. We show analytically how evolutionary dynamics under public goods games in finite adaptive networks can be effectively transformed into a N-Person dilemma involving both coordination and co-existence. Such dynamics would be impossible to foresee from more conventional 2-person interactions as well as from descriptions based on infinite, well-mixed populations. Finally, we show how stochastic effects help rendering cooperation viable, promoting polymorphic configurations in which cooperators prevail.

ew challenges have drawn as much attention from a broad range of disciplines as the evolution of cooperative behaviour. From theoretical biology and behavioural ecology to economics, physics and mathematics, the study of the emergence of pro-social behaviour remains an open and demanding quest, both from experimental and theoretical points of view. In its most general flavour, cooperation dilemmas can be described as a conflict between individual and collective decisions. Take, for instance, the $N$-person Prisoner's dilemma (NPD) - the most common game metaphor for a Public Goods Game (PGG) ${ }^{1-6}$. Here, $N$ players have the opportunity to contribute (or not) a certain amount $c$ to a common pool (the "public good"). The sum of all contributions is invested (that is, it is multiplied by an enhancement factor $F>1$ ) and the return is shared equally among all group members, irrespective of their contribution. From an individual (so-called rational) point of view, defecting or free-riding (not contributing) allows one to rip his/her share of the public good at no cost, to the extent that others help filling in the pot by contributing. Hence, defection emerges as the rational option, leading to the doomsday scenario of the tragedy of the commons ${ }^{2}$, reached when no one contributes and hence there are no resources to share.

Evolutionary game theory (EGT) posits this problem of cooperation by embedding it in a population and defining an evolutionary dynamics within that population of individuals. This formalism provides a natural and intuitive approach to many social and biological eco-systems. Here, the individual game payoff is typically associated with fitness or social success of an individual. The more successful (fitter) individuals will be imitated by others, so that the number of individuals adopting a given behaviour will evolve in time ${ }^{1,7}$. In a well-mixed scenario where each and every individual interacts equally likely with everyone else in the population, and in the absence of additional mechanisms or stochastic effects, a population of Cooperators $(C s)$ and Defectors (Ds) will inevitably evolve towards the extinction of $C$ s. Hence, natural selection leads to the same outcome - the tragedy of the commons - as one expects from a group of rational players. This would happen despite the fact that all players would be better off with overall cooperation.

Several additional mechanisms have been proposed to resolve this paradoxical outcome, from decisions grounded in past encounters, to reputation-based strategies, risk aversion and punishment ${ }^{8-18}$. In particular, it is now well-known that, for both 2-person and $N$-person interactions, the structure of the population may play a determinant role in the evolution of a given behavior ${ }^{6,16,19-29}$. In a networked population, nodes typically represent individuals, whereas links represent shared goals, investments or exchanges. Irrespective of the way our social ties mold the overall population network, it is clear that the well-mixed assumption may only work in the limiting 
cases of small populations, as it is hardly conceivable that, in large populations, everyone can potentially interact with everyone else. Perhaps even more importantly, individuals may have a word on their interacting partners, and with time decide who will be included in their list of peers. In other words, individuals are expected to be able to react against undesired partners, while striving to keep the desired ones, thereby modifying the social network in which they are embedded $^{27,30-39}$. This problem can be addressed considering a dynamical social network. In the following, we study and build up the evolutionary dynamics of cooperation in a population of individuals playing PGGs on a network that may itself evolve as a result of the feedback received from the game interactions.

In a networked context, social neighbourhoods may represent individuals' local universes of partners with whom they can participate in social dilemmas of cooperation. Hence, whenever interacting in an $\mathrm{N}$-person game, individuals will choose their partners from the pool of those they are connected with. In well-mixed populations, in turn, everyone shares the same set of potential partners, which corresponds to the entire population. In this context, well-mixed populations of players interacting in $\mathrm{N}$-person dilemmas can be conveniently viewed as embedded in a fully connected (complete) interaction network (graph).

Let us add to such a setting the possibility that individuals reshape their set of acquaintances. Links may be broken at different rates, likely based on the type of link and its satisfiability ${ }^{6,31,32,40-42}$. Thus, in a world of $C$ s and $D$ s, we have three types of links - CC, $C D$ and $D D$ links - which we may characterize and differentiate, e.g., by attributing different break-up $\left(\gamma_{C C}, \gamma_{D C}, \gamma_{D D}\right)$ and creation $\left(\alpha_{C C}, \alpha_{D C}, \alpha_{D D}\right)$ rates, respectively ${ }^{31,32}$. Depending on the relative values of these rates, and on the composition of the population, different network structures will emerge, with direct impact on each individual's neighbourhoods and ensuing returns from the games they play. These returns will dictate the evolution of strategies in a population which will change its composition, and consequently will influence the network dynamics. In its full scope, this constitutes a tremendously hard problem. In the following section, we shall start by describing in detail a minimal model which, nonetheless, will allow us to gain and analyze key insights of such a co-evolutionary dynamics.

\section{Results}

Let us consider a population of $Z$ individuals, defined as nodes of a dynamical and bidirectional graph. Individuals interact through Public Goods Games (PGGs) played in groups of size N. Individuals setup groups by recruiting members of their neighbourhood (of size $z>N$ ), which thus constitutes the pool of potential co-group players.

The PGG described before has the structure of a NPD, such that the payoff of $C s$ and $D$ s in a group of size $N$ in which $k$ individuals play $C$ and $N-k$ individuals play $D$ can be written as:

$$
\begin{array}{r}
\Pi_{D}(k)=\frac{k F c}{N} \\
\Pi_{C}(k)=\Pi_{D}(k)-c
\end{array}
$$

where $F<N$ ensures a NPD regime.

Let us now address network dynamics. We assume that individuals attempt to form new links at a constant rate $\alpha=\alpha_{C C}=\alpha_{D C}=$ $\alpha_{D D}$. However, the average lifetime of each link will depend on the strategy of the individuals it connects. In this way, we distinguish between $C C, C D$ and $D D$ links. Each of these will be broken at rates $\gamma_{C C}, \gamma_{D C}, \gamma_{D D}$, respectively. This "active linking" dynamics can be conveniently described by the following set of ordinary differential equations ${ }^{31,32}$ :

$$
\dot{N}_{l m}(t)=\alpha\left[\overline{N_{l m}}(t)-N_{l m}(t)\right]-\gamma_{l m} N_{l m}(t)
$$

where $N_{l m}(t)$ is the number of links between individuals of strategy $l$ and $m(\{l, m\} \in\{C, D\})$, and $\overline{N_{l m}}(t)$ is the maximum possible number of these links. This maximum is frequency dependent, in the sense that it will depend on the number of individuals $N_{I}(t)$ and $N_{m}(t)$ playing strategies $l$ and $m$, respectively:

$$
\overline{N_{l m}}(t)=\frac{N_{l}(t)\left[N_{m}(t)-\delta_{l m}\right]}{1+\delta_{l m}}
$$

If the characteristic timescale of the linking dynamics is much shorter than the time scale associated with strategy update (that is, the time that an individual takes to revise his/her strategy), the network will reach a steady state before the next strategy update takes place. That state is characterized by a stationary number of links given by

$$
N_{l m}^{*}=\phi_{l m} \overline{N_{l m}}
$$

where $\phi_{l m}=\alpha\left(\alpha+\gamma_{l m}\right)^{-1}$ represents the fraction of active $l$ - $m$ links.

Assuming that $N_{C}(t)=k$ and $N_{D}(t)=Z-k$ at a given steady state, we can compute the average number of links that any cooperator and defector will have:

$$
\begin{aligned}
& <z_{C}>=\frac{N_{C D}^{*}+2 N_{C C}^{*}}{k} \\
& <z_{D}>=\frac{N_{C D}^{*}+2 N_{D D}^{*}}{Z-k}
\end{aligned}
$$

Substituting (4) in (5) we can express $\left\langle z_{C}\right\rangle$ and $\left\langle z_{D}\right\rangle$ as a function of the fraction of active links:

$$
\begin{aligned}
& <z_{C}>=\phi_{C D}(Z-k)+\phi_{C C}(k-1) \\
& <z_{D}>=\phi_{C D} k+\phi_{D D}(Z-k-1)
\end{aligned}
$$

The model description and calculations performed so far have been carried out in the mean-field approach. In keeping with this spirit, we now calculate the probability of having $j$ cooperators in a group of size $N$, centred on a cooperator $\left(H_{C}(k, j)\right)$ or defector $\left(H_{D}(k, j)\right)$, respectively, whenever the composition of the population is characterized by $k C$ s and $Z-k D$ s. To this end, we employ a hypergeometric sampling ${ }^{10,12,43,44}$ and write:

$$
\begin{aligned}
& H_{C}(k, j)=\left(\begin{array}{c}
<z_{C}> \\
N-1
\end{array}\right)^{-1}\left(\begin{array}{c}
2 N_{C C} / k \\
j
\end{array}\right)\left(\begin{array}{c}
N_{C D} / k \\
N-j-1
\end{array}\right) \\
& H_{D}(k, j)=\left(\begin{array}{c}
<z_{D}> \\
N-1
\end{array}\right)^{-1}\left(\begin{array}{c}
N_{C D} /(Z-k) \\
j
\end{array}\right)\left(\begin{array}{c}
2 N_{D D} /(Z-k) \\
N-j-1
\end{array}\right)
\end{aligned}
$$

From these results we then determine the average fitness of strategies $C$ and $D$ by averaging the payoffs over all possible group compositions:

$$
\begin{aligned}
f_{C}(k) & =\sum_{j=0}^{N-1} H_{C}(k, j) \Pi_{C}(j+1) \\
f_{D}(k) & =\sum_{j=0}^{N-1} H_{D}(k, j) \Pi_{D}(j)
\end{aligned}
$$

Let us now describe how strategies evolve in time. We adopt a stochastic birth-death process combined with the pairwise comparison rule ${ }^{7}$, which accounts for both biological or cultural evolution. In a nutshell, in a biological setting, individuals with higher fitness will reproduce more often; in a cultural evolution setting, individuals with higher returns will tend to be imitated more often. To this end, in each time-step, a randomly chosen individual $i_{1}$ will choose randomly another individual from the population $i_{2}$. The former will imitate the latter with a probability $p$, which increases with the fitness difference. Here we adopt the Fermi distribution, $p=\frac{1}{1+e^{-\beta\left(f_{i_{2}}-f_{i_{1}}\right)}}$, where $\beta$ conveniently specifies the intensity of selection $(\beta \rightarrow 0$ represents neutral drift and $\beta \rightarrow+\infty$ represents pure imitation dynamics $)^{7,45}$. 
In summary, for a finite population of size $Z$, at each birth-death event, there is a certain probability that a cooperator will change into a defector, $T^{-}$, or vice-versa, $T^{+}$; they are given by

$$
T^{ \pm}(k)=\frac{k}{Z} \frac{Z-k}{Z-1} \frac{1}{1+e^{\mp \beta\left(f_{C}(k)-f_{D}(k)\right)}}
$$

whereas the most probable direction of evolution, is given by the so-called gradient of selection, $G(k)=T^{+}(k)-T^{-}(k)$, a finite population analogue of the rate of change of $C s$ stemming from the replicator-like equation ${ }^{46}$ one obtains as $Z \rightarrow \infty$. For finite $Z, G(k)$ reads $^{7}$

$$
G(k)=\frac{k}{Z} \frac{Z-k}{Z-1}\left[\tanh \frac{\beta}{2}\left(f_{C}(k)-f_{D}(k)\right)\right] .
$$

Whenever $G(k)>0(G(k)<0)$, selection will act to increase (decrease) the number of $C$ s.

Gradients of selection. As discussed above, when individuals have enough time to adapt their social ties before deciding whether or not to change their behavioural strategy, then the system of ordinary differential equations which characterizes the linking dynamics may relax towards a steady state. Under such conditions we obtain a closed expression for the average fitness of $C s\left(f_{C}\right)$ and $D s\left(f_{D}\right)$. Clearly, this mean-field approach does not account for the fitness of each particular individual - it confers an average, population-wide, account of the evolution of each behaviour in the presence of a dynamical population structure.

In the absence of any network adaptation mechanism, Ds will benefit from higher returns when compared with $C s$, making the gradient of selection (see eq. (10)) $G(k)<0$, irrespectively of the fraction of $C s(k / Z)$. The same happens whenever links are randomly created and destroyed irrespectively of the strategies of the individuals located at the edges of those links. This can be realized with an adaptive network where $\gamma_{C C}=\gamma_{C D}=\gamma_{D D}$, shown in red in Fig. 1a. Alternatively, we may also consider a more realistic scenario in which the lifetime of a social link depends on the strategy of the individuals it connects. For instance, an individual may be dissatisfied with the return obtained from of her/his partners. This will typically happen whenever an individual is linked to a $D$, in the sense that it is counterproductive to anyone (being him a $C$ or a $D$ ) to be connected (and therefore have him as a potential group member) with a free-rider.

Let us define a scenario, which we shall refer to in the following as Free Choice World (FCW), in which the linking dynamics is characterized by unilateral decisions regarding the break-up of links. In keeping with the previous discussion, this translates into $\gamma_{D D}=\gamma_{C D}$
$>\gamma_{C C}$ or, more conveniently, into $\gamma_{D D}=\gamma_{C D}=\varepsilon \gamma_{C C}$, where $\varepsilon \geq 1$ indicates how fast individuals react when they are linked to a defector. This leads to a different reshaping of the neighbourhoods, compared to the random situation described above, which, in turn, will act to change individuals' fitness. As a result, the impact of strategies on network dynamics leads now to a feedback onto the strategies, by changing their average fitness.

As shown in Fig. 1a, such network adaptation in group interactions creates an evolutionary dynamics scenario corresponding, at a population-wide level, to a new dilemma, characterized by two mixed internal equilibria, which were absent both in well-mixed populations and in the random case (see red line). Furthermore, this modification of the shape of the gradient of selection $G$ transforms cooperation into a viable trait. While both $C s$ (below $k_{L}$ ) and $D s$ (above $k_{R}$ ) become disadvantageous when rare, the co-existence between $C$ s and $D$ s may become a finite population analogue (a probability attractor) of a stable fixed point in infinite populations at a frequency $k_{R} / Z$. Hence, as long as cooperation initially prevails above a certain level $k_{L}$, network adaptation in the framework of the FCW naturally leads to long periods of co-existence between $C$ s and $D s$, rendering cooperation viable.

Let us investigate the roots of $G(k)$. This is equivalent to determining the roots of the fitness difference, $\Gamma(k) \equiv f_{C}(k)-f_{D}(k)$, which, after some algebra becomes:

$$
\begin{gathered}
\Gamma(k)=\frac{F c}{N}\left(\frac{N \phi_{C C}(k-1)+\phi_{C D}(Z-k)}{\phi_{C C}(k-1)+\phi_{C D}(Z-k)}-\right. \\
\left.\frac{(N-1) \phi_{C D} k}{\phi_{C D} k+\phi_{D D}(Z-k-1)}\right)-c
\end{gathered}
$$

Eq. (11) is a second order polynomial in $k$, which means that $\Gamma(k)$ has between zero and two (real) internal roots (in the interval $0<k<$ $Z)$. Their associated stability will be determined by the signs of $\Gamma(1)$ and $\Gamma(Z-1)$ :

$$
\begin{aligned}
\Gamma(1) & =\frac{F c}{N}\left(1+\frac{1-N}{1+(Z-2) / \mu}\right)-c \\
\Gamma(Z-1) & =\frac{F c}{N}\left(1+\frac{1-N}{1+(Z-2) / v}\right)-c
\end{aligned}
$$

Where $\mu=\phi_{C D} / \phi_{D D}$ and $v=\phi_{C D} / \phi_{C C}$.

Given that $Z>N>1$, and whenever $F<N$ - such that individuals play a NPD, it is easy to verify that $\Gamma(1)<0$ and $\Gamma(Z-1)<0$. Thus, we will either have zero or two internal roots, given the order of the polynomial in Eq. (11). In other words we can either have an effective
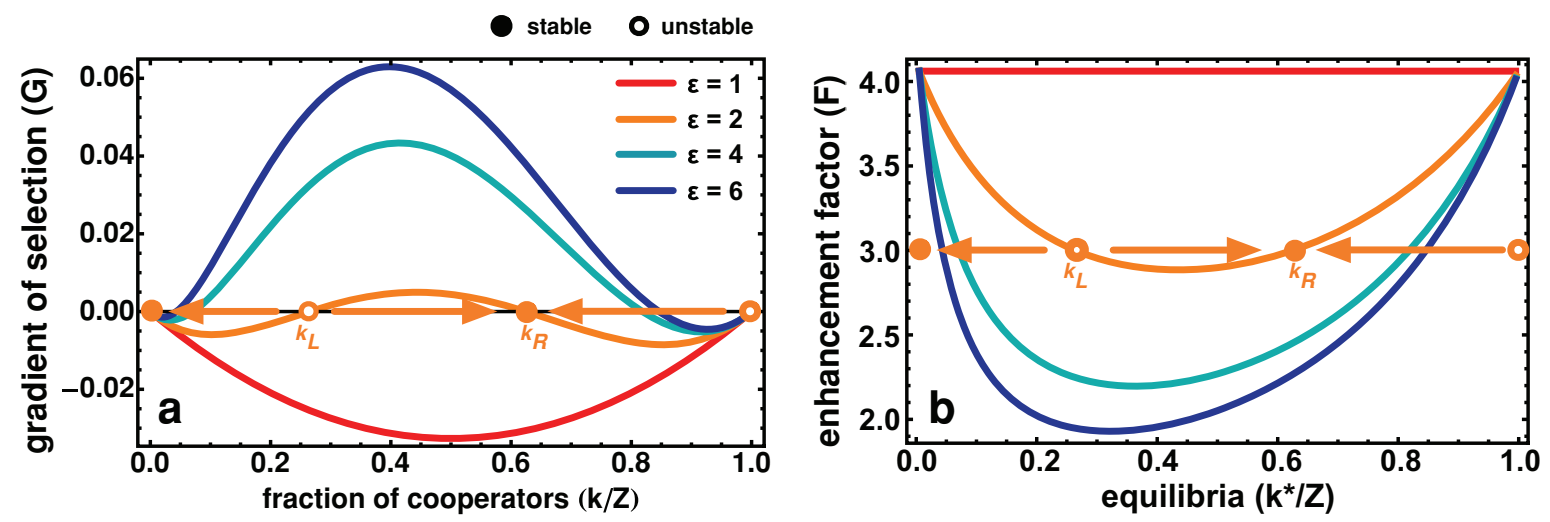

Figure $1 \mid$ Evolutionary dynamics of a NPD in adaptive social structure. We consider a Free Choice World (FCW, see text), grounded on unilateral decision regarding adverse links, such that $\gamma_{\mathrm{DD}}=\gamma_{\mathrm{DC}}=\varepsilon \gamma_{\mathrm{CC}}$. a) Gradient of a selection for various values of $\varepsilon \geq 1$, whereas $Z=200, N=4, F=3, \beta=1$, $\alpha=0.04$ and $\gamma_{C C}=0.1$. Increasing $\varepsilon$ effectively transforms the game, at the population level, from a NPD - where cooperation will always tend to decrease - to a different dilemma characterized by two internal fixed points, typical of an $N$-person coordination game. b) Roots of $G(k)$, as a function of $F$. Above a certain critical value $\left(F>F_{c}\right)$, two internal roots appear that move to the absorbing states as $\varepsilon$ gets larger. 
defection dominance game - negative gradient for all values of $k$ or an $\mathrm{N}$-person coordination dilemma ${ }^{43}$, where the gradient of selection shows both coordination $\left(k_{L}\right)$ and coexistence $\left(k_{R}\right)$ equilibria, with $k_{L}<k_{R}$. Differently, for $F>N$ - where cooperation always prevails in well-mixed populations - the situation will be reversed, that is, $\Gamma(1)>0$ and $\Gamma(Z-1)>0$, in certain cases. For a given $\mu$ and $v$, there is a critical multiplication factor, $F_{c}=N(\omega+Z-2)$ / $[\omega(2-N)+Z-2]$, with $\omega=\max \{\mu, v\}$, above which the gradient will be reversed, i.e., we either have a harmony game or a reversed $N$-person coordination game with $k_{R}<k_{L}$.

The results shown in Fig. 1b confirm these features, and concern the most intereresting case of $F>N$. For each value of $F$, one can draw a horizontal line that defines the location of the internal roots at the intersection with the curves drawn: one unstable root $\left(k_{L}\right)$ and another stable root $\left(k_{L}<k_{R}\right)$. Interestingly, with the increase of the break-up rate $(\varepsilon)$, not only we observe an increase in the critical number of $C s\left(k_{L}\right)$ necessary to reach a stable cooperative basin of attraction, but also we obtain an increase of the stable fraction of $C s$ where the population will spend most of its time, once this basin is reached. Fig. $1 \mathrm{~b}$ also shows that only 2 types of dynamical scenarios can appear: For large multiplication factors $F(F \rightarrow N)$, the above combination involving 2 internal fixed points emerges, whereas the distinctive defection dominance dilemma usually associated with the NPD is recovered for low values of $F$ (as $F \rightarrow 1$ ). It is noteworthy that these results remain valid under a more conventional description of evolutionary dynamics associated with the replicator-like dynamics equation and infinite populations (by taking the limit $Z \rightarrow \infty$, see Eq. (10)) - hence our application of nomenclature (derived from deterministic dynamical systems) to stochastic evolutionary dynamics.

Up to now, link break-up was taken to constitute a unilateral decision. In reality, one can imagine situations in which a connection is broken by mutual consent or as a result of some sort of negotiation towards conflict resolution, which will naturally create an effective break-up rate of links. Specific values will depend on the situation, but in general one may safely assume that $\gamma_{C C}>\gamma_{C D}>\gamma_{D D}$. Hence, in such a Bounded Choice World (BCW), the lifetime of links connecting two Ds will most likely be shorter than the lifetime of links connecting a $C$ and a $D$ (in the sense that it is crucial for a free-rider to have a $C$ in the group, but not additional free-riders). A simple realization of this scenario is obtained assuming, as before $\gamma_{D D}=$ $\varepsilon \gamma_{C C}$, and taking $\gamma_{C D}=\left(\gamma_{C C}+\gamma_{D D}\right) / 2$. As shown in Fig. 2 , this variation with respect to the simpler FCW shown before, does not change the nature of the effective dilemma one obtains at a population level.

Tunneling towards a cooperative basin. Analysis of the gradient of selection provides an overview of the population-wide dynamics. This is particularly relevant, given the coordination barrier, which

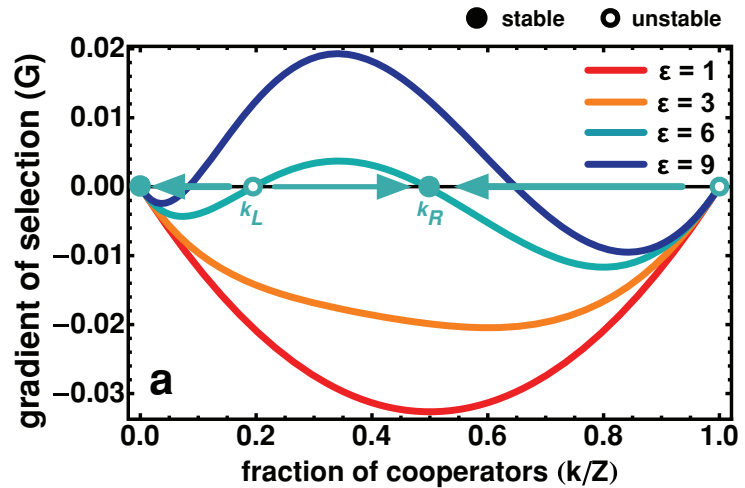

divides the system in two basins of attraction. Classically one uses the fixation probability to calculate the chance of a single $C$ or $D$ to spread and completely invade a population of players using the opposite strategy ${ }^{47}$. However, the existence of stable equilibria may render such an analysis of the fixation probability misleading, as the time required for the population to reach any monomorphic state may become arbitrarily long ${ }^{48}$, in particular whenever $G(k)$ exhibits basins of attraction to stable polymorphic configurations. Furthermore, if one considers other forms of stochastic effects, such as random exploration of strategies ${ }^{49}$ the system will ne3)ver actually fixate.

A convenient alternative which overcomes the drawbacks of the fixation probability, consists in the analysis of the stationary distributions $P(k / Z)^{50}$ of the complete Markov chain (of size $Z+1$ ), defined by the transition probabilities to increase and decrease the number of cooperators. To this end, it is convenient to assume that, besides imitating the actions of the most successful individuals, individuals may explore the available spectrum of strategies with a given (mutation) probability $\mu^{49}$.

The probabilities entering the tridiagonal transition matrix $S=\left[p_{i j}\right]^{T}$ of the Markov chain are defined as $p_{k, k \pm 1}=T_{\mu}^{ \pm}(k)$ and $p_{k, k}=1-p_{k, k-1}-p_{k, k+1}$, where $T_{\mu}^{ \pm}$stand for the transition probabilities computed for an arbitrary mutation probability $\mu$, which are given by $T_{\mu}^{+}(k)=(1-\mu) T^{+}(k)+\mu(Z-k) / Z$ for the probability to increase from $k$ to $k+1 C$ s and $T_{\mu}^{-}(k)=(1-\mu) T^{-}(k)+\mu k / Z$ for the probability to decrease from $k$ to $k-1 C$ s. The stationary distribution is then obtained, as usual, from the eigenvector corresponding to the eigenvalue 1 of $S^{51,52}$

Fig. 3a shows the stationary distribution - i.e., the prevalence in time of a given configuration of the population specified by the fraction of cooperators - for the FCW, adopting the same parameters of Fig. 1 , with $\mu=1 / Z$. As expected, for $\varepsilon=1$, the population spends most of the time in the vicinity of the $k=0$ configuration, as we have a traditional defection dominant dilemma. Yet, as we increase the rate at which individuals react to adverse ties $(\varepsilon)$, we observe the emergence of a single peak near the coexistence equilibrium. Hence, regardless of the initial condition, the population is able to profit from the stochastic effects - resulting both from imitation errors and exploration dynamics - to tunnel through the coordination barrier and spend most of its time at a co-existence configuration in which cooperators prevail.

Finally, as shown in Fig. 3 b, group size $N$ also plays an important role. Using the same parameters of Fig. 3a and setting $\varepsilon=4$, we now vary the group size. For the values used, we always get mixed internal equilibria that move closer to the monomorphic configurations as $N$ gets lower. Qualitatively the picture remains similar to the analysis considered before, in the sense that, as long as we include mutations, the population is able to overcome the coordination barrier and remain most of the time in the configuration associated with the

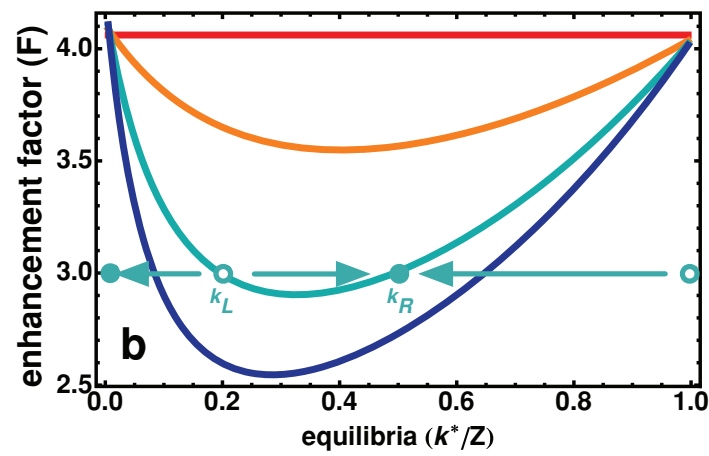

Figure $2 \mid$ Dynamics of a NPD in a Bounded Choice World. a) Gradient of a selection for different values of $\varepsilon \geq 1$, and b) the internal roots of the gradient of selection. Both panels use the same parameters of Fig. 1. 


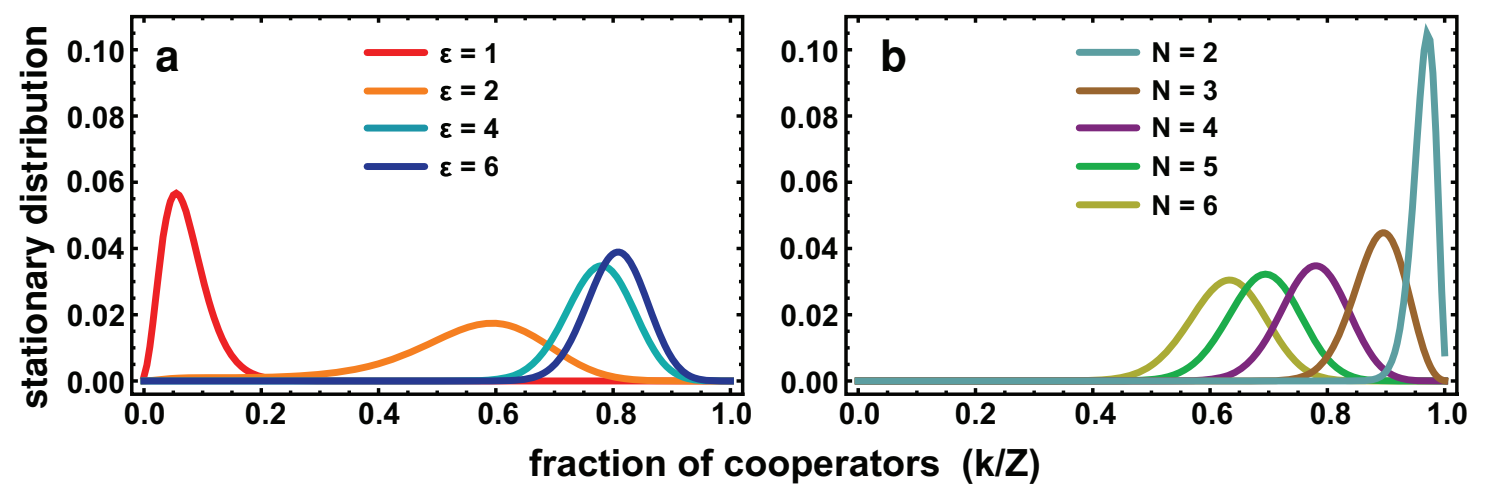

Figure 3 Stationary distribution of cooperators in a FCW. In a) we used the same game parameters as in Fig. 1a and added a mutation probability of $\mu=0.01$. The more prompt individuals are at removing adverse ties (higher $\varepsilon$ ), the easier it is for $C$ s to overcome the coordination barrier. In b) we assess the dependence of the prevailing fraction of cooperation on the group size $N$.

coexistence point. This is particularly relevant, as it shows that, even when we considerably increase the group size (when compared with the population size), network dynamics is able to keep highly cooperative standards, contrary to the conventional wisdom derived from well-mixed populations.

\section{Discussion}

In this paper, we have addressed the evolution of collective cooperative action in dynamical groups, the latter defined by a social network of potential partners that evolve side by side with individuals' behaviour. To do so, we extended a previously developed active-linking analytical framework to $\mathrm{N}$-person dilemmas, which resort to a meanfield description of a network of interactions. Individuals play a Public Goods dilemma and assess the duration of each individual link, which will influence the probability of sharing participation in the same group in the future. Whenever individuals have the chance to promptly modify their universe of partners, the original game at a population level differs from the one at individual level, in this case a NPD. We have shown how the evolution of group composition can affect the evolutionary dynamics of cooperators and defectors transforming a defection dominance dilemma into a new one, characterized by two internal equilibria and two basins of attraction.

This type of dynamics starts to unveil an apparent unifying framework in population dynamics under games of cooperation. Indeed, similar results can be obtained from 2-person dilemmas in static and dynamic social networks ${ }^{53-55}$, to repeated group interactions ${ }^{18}$ and different classes of $\mathrm{N}$-person dilemmas in well-mixed populations, namely those in which individuals face the necessity of some form of prior coordination to achieve a public good ${ }^{16,43,44}$. Similarly, here we have shown how such kind of dynamics is also pervasive when individuals are able to adapt their potential partners in group interactions.

Finally, we have shown how stochastic effects may be determinant in the overall levels of cooperation. This is of particular importance, as stochastic effects may become dominant as a result of errors of different sorts, from behavioural mutations to errors of imitation ${ }^{7,49}$. Indeed, stochastic effects render irrelevant the critical coordination barrier of cooperators needed to reach a stable level of cooperation. In practice, it is as if dynamical networks help escaping the tragedy of the commons by transforming such doomsday scenario into a dilemma in which both strategies can coexist.

1. Sigmund, K. The Calculus of Selfishness. (Princeton University Press, 2010).

2. Hardin, G. The Tragedy of the Commons. Science 162 (3859), 1243-1248 (1968).

3. Kollock, P. Social Dilemmas: The anatomy of cooperation. Annu. Rev. Sociol. 24, 183-214 (1998).

4. Olson, M. The Logic of Collective Action: Public Goods and the Theory of Groups Revised Version ed. (Harvard University Press, 1971).
5. Ostrom, E., Burger, J., Field, C. B., Norgaard, R. B. \& Policansky, D. Revisiting the commons: local lessons, global challenges. Science 284 (5412), 278-282 (1999).

6. Perc, M., Gómez-Gardeñes, J., Szolnoki, A., Floría, L. M. \& Moreno, Y. Evolutionary dynamics of group interactions on structured populations: a review. J. R. Soc. Interface 10 (80), 20120997 (2013).

7. Traulsen, A., Nowak, M. A. \& Pacheco, J. M. Stochastic dynamics of invasion and fixation. Phys Rev E 74 (1), 011909 (2006).

8. Boyd, R. \& Richerson, P. J. The evolution of reciprocity in sizable groups. J. Theo. Biol. 132 (3), 337-356 (1988).

9. Bowles, S. Microeconomics:Behavior, Institutions, and Evolution. (Princeton University Press, 2006)

10. Sigmund, K., De Silva, H., Traulsen, A. \& Hauert, C. Social learning promotes institutions for governing the commons. Nature 466 (7308), 861-863 (2010).

11. Brandt, H., Hauert, C. \& Sigmund, K. Punishing and abstaining for public goods. Proc Natl Acad Sci U S A 103 (2), 495-497 (2006).

12. Hauert, C., Traulsen, A., Brandt, H., Nowak, M. A. \& Sigmund, K. Via Freedom to Coercion: The Emergence of Costly Punishment. Science 316, 1905-1907 (2007).

13. Milinski, M., Semmann, D. \& Krambeck, H. J. Reputation helps solve the 'tragedy of the commons'. Nature 415 (6870), 424-426 (2002).

14. Brandt, H., Hauert, C. \& Sigmund, K. Punishment and reputation in spatial public goods games. Proc. R. Soc. London B 270 (1519), 1099-1104 (2003).

15. Rockenbach, B. \& Milinski, M. The efficient interaction of indirect reciprocity and costly punishment. Nature 444 (7120), 718-723 (2006).

16. Santos, F. C. \& Pacheco, J. M. Risk of collective failure provides an escape from the tragedy of the commons. Proc Natl Acad Sci US A 108 (26), 10421-10425 (2011).

17. Vukov, J., Santos, F. C. \& Pacheco, J. M. Escaping the tragedy of the commons via directed investments. Journal of theoretical biology 287, 37-41 (2011).

18. Van Segbroeck, S., Pacheco, J. M., Lenaerts, T. \& Santos, F. C. Emergence of Fairness in Repeated Group Interactions. Phys Rev Lett 108 (15), 158104 (2012).

19. Szabó, G. \& Hauert, C. Phase transitions and volunteering in spatial public goods games. Phys Rev Lett 89 (11), 118101 (2002).

20. Santos, F. C., Rodrigues, J. F. \& Pacheco, J. M. Graph topology plays a determinant role in the evolution of cooperation. Proc. R. Soc. London B 273 (1582), 51-55 (2006).

21. Szabó, G. \& Fáth, G. Evolutionary games on graphs. Phys Rep 446 (4-6), 97-216 (2007).

22. Ohtsuki, H., Hauert, C., Lieberman, E. \& Nowak, M. A. A simple rule for the evolution of cooperation on graphs and social networks. Nature 441 (7092), 502-505 (2006)

23. Ohtsuki, H. \& Nowak, M. A. The replicator equation on graphs. J. Theor. Biol. 243 (1), 86-97 (2006).

24. Santos, F. C. \& Pacheco, J. M. A new route to the evolution of cooperation. J. Evolution. Biol. 19 (3), 726-733 (2006).

25. Gómez-Gardeñes, J., Campillo, M., Floría, L. \& Moreno, Y. Dynamical organization of cooperation in complex topologies. Phys Rev Lett 98 (10), 108103 (2007).

26. Santos, F. C., Santos, M. D. \& Pacheco, J. M. Social diversity promotes the emergence of cooperation in public goods games. Nature 454 (7201), 213-216 (2008).

27. Perc, M. \& Szolnoki, A. Coevolutionary games--A mini review. BioSystems 99 (2), 109-125 (2010).

28. Santos, F. C., Pinheiro, F. L., Lenaerts, T. \& Pacheco, J. M. The role of diversity in the evolution of cooperation. J. Theor. Biol. 299, 88-96 (2012).

29. Szolnoki, A., Wang, Z. \& Perc, M. Wisdom of groups promotes cooperation in evolutionary social dilemmas. Sci. Rep. 2, 576 (2012).

30. Santos, F. C., Pacheco, J. M. \& Lenaerts, T. Cooperation prevails when individuals adjust their social ties. PLoS Comput Biol 2 (10), e140 (2006). 
31. Pacheco, J. M., Traulsen, A. \& Nowak, M. A. Coevolution of strategy and structure in complex networks with dynamical linking. Phys Rev Lett 97 (25), 258103 (2006).

32. Pacheco, J. M., Traulsen, A. \& Nowak, M. A. Active linking in evolutionary games. J. Theo. Biol. 243 (3), 437-443 (2006).

33. Szolnoki, A., Perc, M. \& Danku, Z. Making new connections towards cooperation in the prisoner's dilemma game. Europhys. Lett. 84, 50007 (2008).

34. Poncela, J., Gómez-Gardeñes, J., Floría, L. M., Sánchez, A. \& Moreno, Y. Complex cooperative networks from evolutionary preferential attachment. PLOS ONE 3 (6), e2449 (2008).

35. Fu, F., Hauert, C., Nowak, M. A. \& Wang, L. Reputation-based partner choice promotes cooperation in social networks. Phys Rev E 78 (2), 026117 (2008).

36. Rockenbach, B. \& Milinski, M. To qualify as a social partner, humans hide severe punishment, although their observed cooperativeness is decisive. Proc Natl Acad Sci U S A 108 (45), 18307-18312 (2011).

37. Fu, F., Wu, T. \& Wang, L. Partner switching stabilizes cooperation in coevolutionary prisoner's dilemma. Phys Rev E 79 (3), 036101 (2009).

38. Fehl, K., van der Post, D. J. \& Semmann, D. Co-evolution of behaviour and social network structure promotes human cooperation. Ecology letters 14 (6), 546-551 (2011).

39. Wang, Z., Szolnoki, A. \& Perc, M. Interdependent network reciprocity in evolutionary games. Sci. Rep. 3, 1183 (2013).

40. Simon, H. A. Reason in human affairs. (Stanford Univ Press, 1983).

41. Van Segbroeck, S., Santos, F., Lenaerts, T. \& Pacheco, J. Reacting differently to adverse ties promotes cooperation in social networks. Phys Rev Lett 102 (5), 58105 (2009).

42. Wu, B. et al. Evolution of cooperation on stochastic dynamical networks. PLoS ONE 5 (6), e11187 (2010).

43. Pacheco, J. M., Santos, F. C., Souza, M. O. \& Skyrms, B. Evolutionary dynamics of collective action in n-person stag hunt dilemmas. Proc R Soc B 276 (1655), 315 (2009).

44. Souza, M. O., Pacheco, J. M. \& Santos, F. C. Evolution of cooperation under N-person snowdrift games. J. Theor. Biol. 260 (4), 581-588 (2009).

45. Szabó, G. \& Toke, C. Evolutionary prisoner's dilemma game on a square lattice. Phys Rev E 58, 69-73 (1998).

46. Hofbauer, J. \& Sigmund, K. Evolutionary Games and Population Dynamics. (Cambridge Univ. Press, Cambridge, UK, 1998).

47. Nowak, M. A., Sasaki, A., Taylor, C. \& Fudenberg, D. Emergence of cooperation and evolutionary stability in finite populations. Nature 428 (6983), 646-650 (2004).

48. Antal, T. \& Scheuring, I. Fixation of strategies for an evolutionary game in finite populations. Bull. Math. Biol. 68, 1923-1944 (2006).
49. Traulsen, A., Hauert, C., De Silva, H., Nowak, M. A. \& Sigmund, K. Exploration dynamics in evolutionary games. Proc Natl Acad Sci U S A 106 (3), 709-712 (2009).

50. van Kampen, N. Stochastic processes in physics and chemistry. (North-Holland, 2007).

51. Imhof, L. A., Fudenberg, D. \& Nowak, M. A. Evolutionary cycles of cooperation and defection. Proc Natl Acad Sci U S A 102 (31), 10797-10800 (2005)

52. Karlin, S. \& Taylor, H. M. A. A first course in Stochastic Processes, 2nd edition ed. (Academic, London, 1975).

53. Van Segbroeck, S., Santos, F. C., Lenaerts, T. \& Pacheco, J. M. Selection pressure transforms the nature of social dilemmas in adaptive networks. New J Phys 13, 013007 (2011).

54. Pinheiro, F. L., Pacheco, J. M. \& Santos, F. C. From Local to Global Dilemmas in Social Networks. PLoS ONE 7 (2), e32114 (2012).

55. Pinheiro, F. L., Santos, F. C. \& Pacheco, J. M. How selection pressure changes the nature of social dilemmas in structured populations. New J Phys 14 (7), 073035 (2012).

\section{Acknowledgments}

This research was supported by FCT-Portugal through grants PTDC/FIS/101248/2008 and PTDC/MAT/122897/2010, by multi-annual funding of CMAF-UL and INESC-ID (under the project PEst-OE/EEI/LA0021/2011) provided by FCT-Portugal. Partial Financial support by the National Science Foundation under Grant No. NSF PHY11-25915 is also gratefully acknowledged.

\section{Author contributions.}

All authors have contributed equally to this work: they all designed and performed the research, analyzed the data and wrote the paper.

\section{Additional information}

Competing financial interests: The authors declare no competing financial interests.

License: This work is licensed under a Creative Commons

Attribution-NonCommercial-NoDerivs 3.0 Unported License. To view a copy of this license, visit http://creativecommons.org/licenses/by-nc-nd/3.0/

How to cite this article: Moreira, J.A., Pacheco, J.M. \& Santos, F.C. Evolution of collective action in adaptive social structures. Sci. Rep. 3, 1521; DOI:10.1038/srep01521 (2013). 